\title{
Protocórtex frente a protomapa: una perspectiva desde el bulbo olfativo
}

\author{
L. López-Mascaraque ${ }^{\text {a }}$, F. de Castro ${ }^{b}$
}

\section{PROTOCORTEX VERSUS PROTOMAP: A PERSPECTIVE FROM THE OLFACTORY BULB}

\begin{abstract}
Summary. Introduction and aim. The olfactory sensory system is a unique model for the research of guidance and connectivity of growing axons. During development, the olfactory epithelium, the olfactory bulb and the olfactory cortex differentiate several cell types and extend projection axons. Because there is a close relationship between these three structures, we ask the question as to whether establishment of the olfactory bulb central projections can proceed independently of the arrival of the olfactory sensory afferents. This raises another more general question: is establishment of afferent connections necessary to awake a developmental program in target cells? Development. The initial establishment of the olfactory bulb central projections occurs independently of the arrival of the olfactory axons from the olfactory epithelium, which reinforces the idea that cortical regions are already patterned before migration of newborn neurons, at least for the olfactory bulb and maybe for the entire brain. This implies a strict intrinsic molecular control of the distinct olfactory structures, independent one of each other. Conclusions. How then, do axonal projections find their correct way within the brain? Contact-mediated mechanisms and chemotropic molecules cooperate to fix their position in the telencephalon, prevent bulbar axons from invading structures other than the olfactory cortex and, at the same time, stimulate axonal branching in an orchestra of both, attractive/promoting and repulsive/inhibiting signals. At later stages, the mature appearance of the olfactory bulb will be completed and refined. [REV NEUROL 2004; 39: 146-55]
\end{abstract}

Key words. Axonal guidance. Development. Mutants. Neurogenesis. Olfaction. Telencephalon.

\section{INTRODUCCIÓN}

Un tema importante en Neurobiología es el efecto que la llegada de las fibras aferentes a sus dianas apropiadas tiene en el desarrollo del sistema nervioso central (SNC), algo que hasta la fecha no se ha aclarado completamente. ¿Dicha llegada desencadena el programa de desarrollo de las células en el sitio que empieza a inervarse o la llegada de las fibras aferentes sirve, simplemente, para refinar los últimos procesos del desarrollo? Con el fin de aclarar esta cuestión, se han propuesto dos teorías para explicar los mecanismos subyacentes a la formación del cerebro. Según el esquema de Rakic [1], existe un 'protomapa', de forma que las diferentes regiones cerebrales se preespecifican antes de la migración de las células posmitóticas (control intrínseco). Al predeterminarse, la especificación se dará por determinantes moleculares [2]. En este modelo, la llegada de los axones aferentes serviría, meramente, para modificar y refinar el protomapa. La segunda teoría es la del 'protocórtex', según la cual las diferentes áreas corticales provienen de una corteza indiferenciada que se especializa como resultado de las aferencias talámicas (control extrínseco) [3].

Actualmente, es de gran interés el hecho de que una o ambas hipótesis puedan también operar durante el desarrollo del sistema olfativo. La naturaleza de las interacciones entre el epitelio olfativo (EO), el bulbo olfativo (BO) y la corteza olfati-

Recibido: 16.06.04. Aceptado tras revisión externa sin modificaciones: 17.06.04.

${ }^{a}$ Instituto Cajal-CSIC. Madrid. ${ }^{b}$ Instituto de Neurociencias de Castilla y León (INCyL). Universidad de Salamanca. Salamanca, España.

Correspondencia: Dra. Laura López-Mascaraque. Instituto Cajal-CSIC. Avda. Dr. Arce, 37.E-28002 Madrid. Fax: +34915854 754.E-mail: mascaraque@ cajal.csic.es

Proyecto de LLM: BFI 2003-00139 (MCYT). Proyectos de F de C: PI020768 (FIS, Ministerio de Sanidad), SA053/04 (Junta de Castilla y León) y Fundació la Caixa.

(C) 2004, REVISTA DE NEUROLOGÍA va $(\mathrm{CO})$ en las etapas precoces del desarrollo es un campo abonado, a día de hoy, para el estudio [4,5]. Con la idea de ahondar en el conocimiento de los mecanismos involucrados en la especificación y el desarrollo del sistema olfativo, esta revisión trata de los diferentes estudios de Neurobiología del desarrollo. Nuestros estudios en el ratón mutante para Pax6 nos llevaron a la conclusión de que el inicio de la formación de las proyecciones centrales del $\mathrm{BO}$ puede ocurrir de forma completamente independiente a la llegada de los axones sensoriales desde el EO [6]. Como observaremos en este trabajo, los más recientes estudios parecen corroborar nuestra hipótesis. Este hecho nos lleva a plantear si, durante el desarrollo, podemos considerar al BO como un dominio independiente, lo que corroboraría la hipótesis del protomapa. Asimismo, discutiremos también la orquesta molecular involucrada en la formación de las proyecciones desde el BO hasta la CO.

\section{BREVE REPASO DEL SISTEMA OLFATIVO}

El establecimiento de los circuitos básicos en el sistema olfativo de los mamíferos comienza con las neuronas sensoriales olfativas (NSO), o receptores olfativos, localizados en el EO, estructura que se desarrolla durante la embriogénesis a partir de la placoda olfativa. Los axones de las NSO proyectan al BO, donde establecen contactos sinápticos con las dendritas de las células mitrales y empenachadas y, junto con las células periglomerulares, dan lugar a los glomérulos del BO [7,8]. Cada uno de los NSO -en roedores hay aproximadamente cinco millones, distribuidos por toda la cavidad nasal- expresa, solamente, uno de los 1.000-1.300 genes específicos de los receptores olfativos que se conocen $[9,10]$. Las NSO que expresan un mismo gen de receptor odorífero se distribuyen de forma aleatoria dentro de la cavidad nasal; pero, sus axones proyectan de forma selectiva sobre 1-4 glomérulos de los aproximadamente 2.000 que existen en el BO -para una revisión, ver Mombaerts et al [11]-. Los axones primarios de las neuronas 


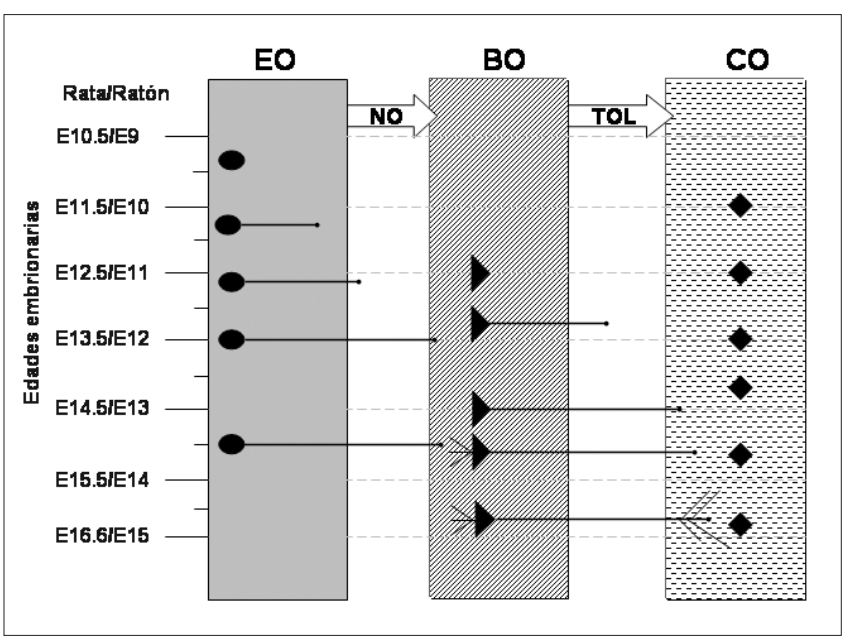

Figura 1. Representación esquemática de la neurogénesis y formación de axones en el sistema olfativo. La génesis de las NSO (óvalos) empieza alrededor de E9-E9,5, la de las células mitrales (triángulos) alrededor de E11 y en la corteza olfativa alrededor de E10 (rombos). La formación de los axones de las células mitrales $(E 11,5)$ comienza antes de la llegada de los axones del NO a la superficie telencefálica (E12). Las células mitrales comienzan a emitir dendritas en el momento que los primeros axones sensoriales entran en el BO $(E 13,5)$. Los axones del TOL forman colaterales que invaden sus dianas corticales en E14,5-15. La génesis neuronal en los tres compartimentos del sistema olfativo empieza de forma independiente, presumiblemente debido a mecanismos de control interno (hipótesis del protomapa). Todas las edades referidas en este pie de página corresponden a ratón (ver equivalentes en la rata a la izquierda del esquema). En la figura hemos referido datos de diferentes autores [6,16,27,33-40]. En una escala rata/ratón la fecha de detección del tapón vaginal en la madre es E0. TOL: tracto olfativo lateral; BO: bulbo olfativo; NO: nervio olfativo; NSO: neuronas sensoriales olfativas (modificado de [107])

de proyección del BO (mitrales y empenachadas), crecen preferentemente a lo largo de una zona muy estrecha del telencéfalo ventrolateral, adyacente a la superficie pial. En esa región forman el tracto olfativo lateral (TOL) [7,12-17]. De los axones del TOL se ramifican colaterales que colonizan, en un preciso orden rostrocaudal, las regiones diana de la $\mathrm{CO}$ : el núcleo olfativo anterior, el córtex piriforme, el tubérculo olfativo, la corteza entorrinal y diversos núcleos amigdalinos [14,15,1820]. Estudios recientes han demostrado que existe un mapa topográfico en las aferencias del BO a la CO. Las aferencias de las NSO que expresan el mismo receptor odorífero proyectan -vía BO- a grupos neuronas diana en la corteza. Esta disposición permite integrar cada uno de los códigos específicos de los receptores odoríferos, lo que deja generar sensaciones odoríferas complejas y, subsiguientemente, transmitir dicha información a otras áreas corticales [21]. La laminación definitiva del BO depende de la llegada de las células que migran de forma tangencial desde la zona subventricular del cerebro anterior, las interneuronas prospectivas del $\mathrm{BO}$-revisado por Goldman y Luskin [22].

Después de esta breve introducción al sistema olfativo, los dos esquemas de desarrollo que hemos citado anteriormente podrían sugerir muy diferentes rutas. Mientras la teoría del protomapa predice una organización inicial, simultánea e independiente, del EO, del $\mathrm{BO}$ y de la $\mathrm{CO}$, la teoría del protocórtex predice que el desarrollo del $\mathrm{BO}$ sería un proceso secundario, indirecto, dependiente de la llegada de los axones de las NSO desde el EO. Según este modelo, la llegada subsiguiente de las fibras del TOL al córtex induciría, necesariamente, la diferenciación celular en esta estructura.

\section{INFLUENCIAS MUTUAS ENTRE EL EPITELIO OLFATIVO Y EL BULBO OLFATIVO}

La influencia del EO en poner en marcha el desarrollo del BO es algo demostrado en anfibios [23-26]. Sin embargo, esta relación se estudia con detenimiento en mamíferos en los últimos años. Por ejemplo, se ha observado que la llegada de los axones olfativos parece tener una influencia dramática en la cinética de las células y en la velocidad de diferenciación de los neuroblastos en el anlaje del BO [27]. Se ha sugerido, también, que interacciones de tipo inductivo del mesénquima frontonasal son esenciales para la morfogénesis tanto del EO como del BO $[5,28]$. De cualquier forma, estos trabajos no se han extendido a etapas tempranas de la formación de la placoda olfativa [5].

En las etapas iniciales del desarrollo, la parte dorsorrostral del telencéfalo de los mamíferos se especifica ya como primordio del BO [16,29-31]. Dicho anlaje expresa Pax-6 [31,32]. La llegada de los axones de las NSO induce la evaginación macroscópica del $\mathrm{BO}$ y su laminación prenatal; pero, en ningún caso induce la neurogénesis de las neuronas mitrales $[31,33,34]$. De hecho, las células mitrales se han generado antes de la llegada de los citados axones [16,35]. Las NSO empiezan a expresar la OMP (del inglés, olfactory marker protein) en E16, en la rata (E14-14,5 en el ratón), bastante después de la formación de las primeras conexiones entre el EO y el BO [36-39]. Pero, no se determina el papel inductivo que desempeña el BO en este tipo de maduración. En el mutante homocigoto para el gen Pax-6 comienza a desarrollarse un BO defectivo -denominado OBLS, del inglés olfactory bulb like structure- en la vesícula telencefálica, en total ausencia de un nervio olfativo (NO) [6,31]. La posición ectópica del OBLS se debe a un fallo en la migración celular, no a un tipo de neurogénesis ectópica, ya que las células que remedan el fenotipo mitral en el OBLS se generan en el mismo lugar que en ratones control (telencéfalo dorsorrostral: 31), emiten sus axones y forman un tracto reminiscente del TOL (ver más detalles más adelante). Estas observaciones sugieren que la hipótesis del protomapa debería aplicarse a los eventos más tempranos del desarrollo del sistema olfativo - p. ej., la neurogénesis de las células mitrales y empenachadas en el primordio del BO y la neurogénesis de las NSO en la placoda olfativa, con sus respectivas proyecciones eferentes-. En cualquier caso, múltiples interacciones inductivas tejido-tejido [5] se implicarían en los eventos sucesivos que ocurren en el desarrollo - p. ej., la evaginación macroscópica y la formación de las capas en el BO-. Esto se basa en que la neurogénesis esta desfasada entre el BO y el EO: la producción de células mitrales se inicia en E10-10,5 en el BO del ratón, mientras las NSO del EO se generan a partir de E9,5 en el EO del ratón (ratón: [6,39,40]; rata: [33-36]) (Fig. 1). En las diferentes áreas de la CO, la neurogénesis ocurre en una sucesión anteroposterior, y empieza bastante antes de la llegada de los primeros axones procedentes de las neuronas de proyección del BO [18,41] (Fig. 1). Recientemente, se ha sugerido que los glomérulos supernumerarios del BO no se estabilizan porque el número de axones aferentes escasea para poder mantener una formación estable de los mismos. A esto se le ha denominado 'fenómeno de interdependencia' entre los axones de las NSO [42]. En la figura 1 mostramos un sumario comparativo, entre el ratón y la rata, de la escala temporal de generación de las neuronas y la elongación axonal, para los tres compartimentos principales del sistema olfativo. En los ratones, las primeras NSO se generan en E9, mientras los primeros axones de las NSO se diferencian en E9,5-E10,5. Estos axones no 
alcanzan a la vesícula telencefálica/primordio del BO antes de E11, medio día más tarde de que aparezcan las primeras células mitrales (E10,5). Los primeros axones mitrales en abandonar el BO se observan en E11,5 y empiezan a inervar la CO en E15, bastante después de que se inicie la neurogénesis en el córtex piriforme (E9,5-E11).

De acuerdo con la hipótesis del protocórtex, ¿podríamos imaginar que el EO y el BO se desarrollan de forma independiente? La hipótesis del protocórtex se basa en una secuencia de eventos, dentro de la que se adivina una especie de jerarquía. En esta jerarquía, el EO prevalecería sobre el BO: el EO dictaría el desarrollo del $\mathrm{BO}$ desde el primer momento, tal y como han sugerido algunos autores [26,27,43]. Pero, ninguno de estos científicos ha propuesto que exista una relación similar entre el BO y el córtex olfativo, quizás por la existencia de reconocidos estudios acerca del inicio de la neurogénesis en la corteza $[18,39,41]$. ¿Acaso no debemos incluir estos experimentos en el contexto del desarrollo del sistema nervioso o cuando se trata de argüir a favor de una secuencia de eventos entre el EO y el BO? Uno de los puntos de apoyo primordiales de la hipótesis del protocórtex es el hecho de que la génesis de las células mitrales empieza en E11 en el ratón [39], mientras la rata comienza en E14 en el caso de La Mantia et al [27]. Si nos basamos en la escala temporal que se representa en la figura 1 , podríamos esperar que la producción de células mitrales comenzase en E12,5 en la rata, y no en E14. Esto implica que la edad en que estos autores consideran que los primeros axones de las NSO entran en la vesícula telencefálica, resulta que ya ha comenzado la neurogénesis en el anlaje del BO. Así que, la secuencia de eventos EO-BO propuesta sólo se basa en diferencias en la forma de determinar las etapas del desarrollo embrionario. Una independencia equivalente es la que se observa en el caso de la CO (Fig. 1), donde las neuronas corticales se generan con bastante antelación a la llegada de los primeros axones mitrales.

Déjenos el lector volver su atención hacia los resultados de diferentes modelos experimentales en los que, bien el $\mathrm{BO}$, bien el EO, se han alterado. Las neuronas de proyección del BO expresan fuertemente el homólogo en los mamíferos del gen brachiury, que es el gen Tbrl. En mutantes nulos para Tbrl sobreviven unas pocas células con fenotipo mitral, que no proyectan al córtex olfativo (ver más adelante). Por el contrario, y sin importar para ello la ausencia de sus dianas -las dendritas de las células mitrales-, los axones de las NSO convergen normalmente en el BO, y forman estructuras esféricas que recuerdan a los glomérulos [29] (Tabla). Paralelamente, en mutantes nulos para los genes homeobox de mamífero, $D l x-1$ y $D l x$-2, se ha demostrado la ausencia de interneuronas [29] (Tabla); sin embargo; los axones olfativos convergen para dar lugar a esferas glomerulares, lo que sugiere que éstas tampoco contribuyen de forma significativa a la hora de determinar las dianas de las NSO [29] (Tabla). Al contrario, en mutaciones nulas del gen homeobox Emx-2 -pero no en el Emx-1-, el NO no llega a alcanzar el BO. Como resultado de ello, la capa de células mitrales se desorganiza y, quizás sorprendentemente, se forma un TOL aparentemente normal [44] (Tabla). Una ausencia de laminación característica del BO se observa en una mutación en el gen $D l x-5$, que provoca la falta de NSO que contacten con el OB [45]. En el caso de ratones mutantes en el gen Mash-1, que se expresa en progenitores neurales que igualmente carecen de la conexión EO-BO [46,47], produce efectos similares a la mutación en el gen $D l x-5$, con inclusión de una drástica reducción en el número de NSO. De hecho, para algunos autores, la especificación de las células mitrales ocurriría de forma totalmente independiente de la inervación por parte de los axones olfativos [31,45]. Los datos obtenidos en los mutantes nulos de Pax-6, Tbr-1, Dlx-1, Dlx-2, Dlx-5, Mash-1 y Emx-2 (Tabla) argumentan a favor del establecimiento de un mapa topográfico de conexiones entre el EO y el territorio presuntivo del OB, independientemente de la presencia de neuronas de proyección e interneuronas y, evidentemente, de los posibles factores que ambas puedan aportar. En cualquier caso, los datos del mutante de Emx-2 no sugieren que los axones de las NSO ayuden a asegurar la disposición ordenada de las células mitrales, aunque la significación funcional de este extremo no está clara. Un caso singular es el del receptor de baja afinidad para neurotrofinas, p75NTR, que influye, de forma indirecta en el crecimiento de los axones olfativos -en el período posnatal temprano hay un crecimiento exuberante de algunos axones de las NSO-, pero su convergencia en el BO es normal y originan, transitoriamente, una laminación anormal en la porción dorsocaudal del BO [48].

¿Qué ocurre tras la ablación genética o quirúrgica del EO? La expresión de receptores odoríferos a cargo de las NSO es completamente independiente del $\mathrm{BO}$, ya sea en ausencia del BO durante la embriogénesis [49] o durante la regeneración tras la axotomía de las NSO [50,51]. La deprivación sensorial olfativa en neonatos, ya sea total o parcial-oclusión de las coanas por electrocauterización-, aparentemente, no tiene efecto en el número de NSO presentes en el EO [52] (Tabla). De forma similar, y aunque la deprivación sensorial comporta una reducción del $25 \%$ en el tamaño del BO, las poblaciones celulares se estructuran de forma correcta y en números normales [52-57] (Tabla). Aún así, Meisami y Safari [58] dan testimonio de una mayor pérdida en el número de células empenachadas que en el de mitrales tras la deprivación olfativa. Ni el número ni el tamaño de los glomérulos parecen cambiar tras dicha manipulación, pero observan un aumento de la razón células mitrales/células granulares [52]. Es más, el patrón de reinervación que se observa en las NSO generadas tras una deaferentación química global es muy parecido a la topografía original [59]. En suma, la oclusión de las narinas parece tener sus mayores efectos tempranos tras el nacimiento, aunque hay que tener en cuenta que dicho procedimiento es extremadamente difícil de llevar a cabo antes del nacimiento y no se ha realizado en el embrión [26]. En general, la deprivación se realizó en individuos posnatales/adultos, cuando todas las conexiones entre el EO y el BO ya se han establecido. En cualquier caso, se han observado cambios similares cuando la señalización odorífera se ha interrumpido genéticamente antes de que se establezcan las citadas conexiones, por ejemplo, en animales sin canales de compuerta de nucleótidos cíclicos $-\mathrm{G}_{\text {(olf) }}$, la mayor subunidad $\alpha$ de la proteína $\mathrm{G}$ en las NSO- $[60,61]$ (Tabla) o sin OMP, que participa de la percepción odorífera [62] (Tabla). Es más, algunas proyecciones olfativas periféricas se afectan en mutantes nulos para una subunidad del canal de compuerta de nucleótidos cíclicos, lo que sugiere que la navegación de estos axones se influye, al menos en parte, por la actividad odorífera [63]. Esto es algo especialmente relevante para las células mitrales, cuya única respuesta a la oclusión de las narinas se refiere al tamaño del perikarion y a las sinapsis recíprocas entre células mitrales y granulares, mientras que el área de sección del TOL sigue completamente normal [52]. No se observan cambios hipertróficos en las láminas 
Tabla. Influencia del epitelio olfativo (EO) en el bulbo olfativo (BO). Aproximaciones experimentales que incluyen lesiones físicas, químicas o funcionales. Resultados de los diferentes modelos experimentales en los que se ha alterado el bulbo olfativo y/o el epitelio olfativo (modificado de [107]).

\begin{tabular}{|c|c|c|c|c|c|c|c|}
\hline & Animal & NSO & NO & $\begin{array}{l}\text { Convergencia } \\
\text { NO en BO }\end{array}$ & $\begin{array}{l}\text { Células mitrales/ } \\
\text { empenachadas }\end{array}$ & $\begin{array}{l}\text { Interneuronas } \\
\text { del BO }\end{array}$ & TOL \\
\hline \multicolumn{8}{|l|}{ Axotomía y destrucción química } \\
\hline Ablación de PO [24] & Embriones de Xenopus & $\varnothing$ & $\varnothing$ & $\varnothing$ & $\varnothing$ & $\varnothing$ & $\varnothing$ \\
\hline Axotomía (parcial) de NO [25] & Embriones de Xenopus & $\downarrow \quad \downarrow(50 \%)$ & $\downarrow(50 \%)$ & $?$ & $\downarrow(50 \%)$ & $?$ & $?$ \\
\hline Ablación de BO [50] & Rata adulta & N & $\varnothing$ & $\varnothing$ & N & $\mathrm{N}$ & N \\
\hline Ablación de BO [51] & $\begin{array}{l}\text { Pez gato joven } \\
\text { (regenerado) }\end{array}$ & $\stackrel{\mathrm{N}}{\text { (regenerado) }}$ & $\varnothing$ & $\varnothing$ & $\varnothing$ & $\varnothing$ & $\varnothing$ \\
\hline \multicolumn{8}{|c|}{ Privación olfatoria total/parcial posnatal } \\
\hline Oclusión orificio nasal [54] & Rata posnatal & $?$ & $?$ & $?$ & $\begin{array}{c}\mathrm{N} \\
\text { (BO pequeño) }\end{array}$ & $\begin{array}{c}\mathrm{N} \\
\text { (BO pequeño) }\end{array}$ & N \\
\hline $\begin{array}{l}\text { Cierre orificio nasal: } \\
\text { electrocauterización [52] }\end{array}$ & Ratón neonato & $\mathrm{N}$ & N & $\mathrm{N}$ & $\begin{array}{c}\mathrm{N} \\
\text { (BO pequeño) }\end{array}$ & N & N \\
\hline $\begin{array}{l}\text { Privación de estímulo: } \\
\text { cierre de un orificio nasal [54] }\end{array}$ & Ratas P25 & $?$ & $?$ & $?$ & $\begin{array}{c}\downarrow(40 \%) \\
\text { (BO pequeño) }\end{array}$ & $?$ & $?$ \\
\hline $\begin{array}{l}\text { Cierre quirúrgico } \\
\text { del orificio nasal [56] }\end{array}$ & Ratas P1-P30 & $?$ & $?$ & $?$ & $\begin{array}{c}\mathrm{N} \\
\text { (BO pequeño) }\end{array}$ & $\begin{array}{c}\uparrow \\
\text { (BO pequeño) }\end{array}$ & $\mathrm{N}$ \\
\hline Denervación focal [55] & Ratas $\mathrm{P} 10$ y $\mathrm{P} 20$ & $?$ & $\downarrow$ (focal) & $?$ & $\downarrow$ & $?$ & N \\
\hline Lesión química [59] & $\begin{array}{l}\text { Ratón transgénico } \\
\text { H-OMP-lacZ-6 }\end{array}$ & $\begin{array}{c}\mathrm{N} \\
\text { (recuperado) }\end{array}$ & $\begin{array}{c}\mathrm{N} \\
\text { (recuperado) }\end{array}$ & $\begin{array}{c}\mathrm{N} \\
\text { (recuperado) }\end{array}$ & $\mathrm{N}$ & N & $?$ \\
\hline \multicolumn{8}{|l|}{ Deprivación funcional } \\
\hline $\mathrm{G}_{\text {olf }}{ }^{-1-}[61]$ & Ratón neonato & $N^{*}$ & $\mathrm{~N}$ & $\mathrm{~N}$ & $\mathrm{~N}$ & $?$ & $?$ \\
\hline$O_{\text {cng }^{-}}$channel $^{-1-}[60]$ & Ratón & $\mathrm{N}^{*}$ & $\mathrm{~N}$ & N & N (BO pequeño) & $\mathrm{N}$ & N \\
\hline Mutagénesis gen OCNC [63] & Ratón & $\begin{array}{l}\mathrm{N}^{*} \\
\text { (NOS inactivas) }\end{array}$ & $\mathrm{N}$ & $\mathrm{N}$ & $?$ & $?$ & $?$ \\
\hline \multicolumn{8}{|l|}{ Animales mutantes } \\
\hline${\operatorname{Pax}-6^{-1-}[6]}$ & $\begin{array}{l}\text { Embriones } \\
\text { de ratón Sey }\end{array}$ & $\varnothing$ & $\varnothing$ & $\varnothing$ & $\begin{array}{c}\text { Células similares } \\
\text { a mitrales en } \\
\text { OBLS }\end{array}$ & Desorganizado & $\mathrm{N}$ \\
\hline$E m \times 2^{-1-}[44]$ & Embriones de ratón & $N ?$ & $N ?$ & $\varnothing$ & Desorganizado & $\mathrm{N}$ & $\mathrm{N}$ \\
\hline$X t^{J-1-}[49]$ & $\begin{array}{l}\text { Embriones de ratón } \\
\text { (agénesis del } \mathrm{BO} \text { ) }\end{array}$ & $\mathrm{N}$ & $\mathrm{N}$ & $\varnothing$ & $\varnothing$ & $\varnothing$ & $\varnothing$ \\
\hline Tbr1 ${ }^{-1-}$ [29] & Embriones de ratón & $\mathrm{N}$ & $\mathrm{N}$ & $\mathrm{N}$ & $\varnothing$ & Desorganizado & $\varnothing$ \\
\hline$D / \times 1^{-1-}, D / \times 2^{-/-}[29]$ & Embriones de ratón & N & N & N & N & $\varnothing$ & $?$ \\
\hline NCAM-180-/- [78] & Embriones de ratón & $\mathrm{N}$ & $\mathrm{N}$ & $\mathrm{N}$ & $\begin{array}{c}\mathrm{N} \\
\text { (BO pequeño) }\end{array}$ & $\begin{array}{c}\mathrm{N} \\
\text { (BO pequeño) }\end{array}$ & $\mathrm{N}$ \\
\hline$O M P^{-/}[62]$ & Ratón posnatal & $N$ & $\mathrm{~N}$ & $\mathrm{~N}$ & $N$ & $N$ & $?$ \\
\hline p75NTR ${ }^{-1-}[48]$ & $\begin{array}{l}\text { Embriones de ratón } \\
\text { y posnatales }\end{array}$ & $\mathrm{N}$ & N & $\mathrm{N}$ & $\stackrel{\mathrm{N}}{\text { (BO evaginado) }}$ & $\begin{array}{c}\mathrm{N} \\
\text { (BO evaginado) }\end{array}$ & $\mathrm{N}$ \\
\hline Kall-1 [106] & Humanos & $?$ & $?$ & $\varnothing$ & $\begin{array}{c}\text { (Aplasia/ } \\
\text { hipoplasia BO) }\end{array}$ & $\begin{array}{c}\text { (Aplasia/ } \\
\text { hipoplasia BO) }\end{array}$ & $\varnothing$ \\
\hline$D / \times 5^{-1-}[45]$ & Embriones de ratón & $\begin{array}{c}\text { No emiten } \\
\text { axones } \\
\text { (EO pequeño) }\end{array}$ & $\varnothing$ & $\varnothing$ & $\begin{array}{c}\text { Alteración en } \\
\text { orientación }\end{array}$ & $\begin{array}{c}\downarrow \\
\text { (Desorganizado) }\end{array}$ & $\bar{N}$ \\
\hline Mash1 $^{-1-}[46,47]$ & Embriones de ratón & $\varnothing$ & $\varnothing$ & $\varnothing$ & $\begin{array}{c}\mathrm{N} \\
\text { (BO pequeño) }\end{array}$ & $\downarrow$ & $\mathrm{N}$ \\
\hline
\end{tabular}


de los BO que no se han deprivado, lo que indica que no hay cambios compensatorios tras la deprivación olfativa [52].

Resulta interesante que el bloqueo de la transducción odorífera, ya sea por oclusión de las narinas, ya por ingeniería genética encaminada a la pérdida de receptores o de canales, no afecta en ningún caso a la convergencia de los axones de las NSO en el BO [60,61] (Tabla). Es más, la proyección desde el EO se restaura tras la ablación genética, lo que sugiere que las señales de posición involucradas en la formación del NO deben persistir en el BO a lo largo de toda la vida del individuo [64]. Así, no parece que exista un putativo mecanismo 'pionero' para el resto de las fibras olfativas, aunque deberían hacerse estudios de alta resolución de este aspecto [64,65]. En conjunto, esto puede explicar el hecho de que las NSO que se generan de forma continua a lo largo de la vida de un individuo establezcan las conexiones correctas dentro del BO, también a todo lo largo de la existencia del animal, y que el mapa de proyecciones olfativas permanezca estable, sin que le afecte la mencionada renovación constante en la población de NSO.

Es algo comúnmente aceptado que las diferentes mutaciones del gen Pax-6, con inclusión de la mutación espontánea denominada Small Eye [66,67], producen diferentes alteraciones. Entre ellas, la ausencia de ojos y de estructuras nasales, tales como el EO y el BO. Con la utilización de una batería de marcadores moleculares región y campoespecíficos, un estudio detenido de estos mutantes revela la presencia de un BO prospectivo en la zona rostrolateral de la vesícula telencefálica, denominada OBLS [6,31] (Tabla). Nuestros hallazgos sugieren que la mutación en el gen Pax-6 trastorna el desarrollo anatómico, sin alterar la diferenciación del BO y el inicio de la formación de su proyección central. Hasta la fecha, hemos sido incapaces de identificar un NO que entre en el OBLS de estos mutantes [6]. Por desgracia, el mutante PCD - un ratón que, de forma espontánea, tiene un déficit de células de Purkinje-, en el que se ha demostrado una pérdida total de células mitrales, no es un modelo complementario adecuado, ya que la pérdida de células mitrales ocurre a los 2-4 meses de edad, mientras que las células empenachadas permanecen normales. Además, el número de células en el BO es normal, tanto en el caso del embrión como en el adulto joven de los mutantes PCD [68]. En cualquier caso, el grosor del TOL en los animales posnatales es significativamente menor tras la pérdida de las células mitrales.

En suma, todos estos estudios demuestran que las células del BO pueden sobrevivir sin EO; incluso pueden empezar a diferenciarse sin la llegada de sus aferentes primarias. Es más, las NSO son capaces de sobrevivir y de continuar con su constante reemplazo y regeneración axonal incluso en ausencia de $\mathrm{BO}$, aunque la velocidad del reemplazo puede afectarse. En cualquier caso, ambas estructuras parecen ser independientes, la una de la otra, para su respectiva supervivencia. Estas observaciones no contradicen las realizadas por otros autores acerca de la influencia de los axones de las NSO en etapas más tardías del desarrollo del BO [16,23,27,38,57].

\section{SEÑALES MOLECULARES QUE GUÍAN LA FORMACIÓN DEL TOL}

Si la formación de las distintas estructuras que componen el sistema olfativo y sus conexiones axónicas progresan, inicialmente, de forma independiente, la identificación de los factores que controlan ambos procesos debería arrojar alguna luz sobre algu- no de los puntos más controvertidos que revisamos con este trabajo. Es posible que los axones de las neuronas de proyección del BOA, localizados en las zonas más profundas del TOL, puedan servir como axones pioneros para, subsiguientemente, guiar los axones del BO. El proceso temporal de la formación del TOL y de la llegada de los axones de las células mitrales a sus dianas en la CO apoya esta idea $[15,16,35]$. Aunque no hay que olvidar que, en ausencia de los axones del BOA, los de las células mitrales del BO entran y forman un TOL [40]. Esta observación parece excluir la idea de que las neuronas de proyección del BOA envíen axones pioneros, sino que, más bien, sugiere que hay señales intrínsecas del telencéfalo que serían las responsables de la formación del TOL [6]. A continuación, analizaremos en detalle dos de los mecanismos más plausibles.

\section{Mecanismos por contacto}

Las moléculas de adhesión celular son buenos candidatos para ser señales por contacto válidas a la hora de formarse el TOL; particularmente algunas, como NCAM-H [69], OCAM y NrCAM. Estas últimas podrían actuar en conjunto o en combinación con TAG-1/axonin-1 [70], aunque, hasta la fecha, ninguna se ha implicado directamente en la formación del TOL. Tanto laminina como -y aunque en menor medida- fibronectina promueven el crecimiento in vitro de los axones de las neuronas de proyección del $\mathrm{BO}$ y también podrían tener un efecto relevante in vivo [71]. Otras moléculas que actúan por contacto, tan relevantes como la reelina o las ephrinas/Eph, no parece que desempeñen ningún papel en la formación del TOL [72,73]. La Sema 7A -un miembro de la familia de las semaforinas que se ancla a membrana por medio de un residuo GPI- se ha implicado en la formación del TOL y, aunque no influya en la orientación del crecimiento de los axones mitrales, ni tampoco en la formación de sus colaterales, sí podría ser el primer factor involucrado, directamente, en el control del crecimiento en sentido rostrocaudal de los axones del TOL [74]. También se ha propuesto la existencia de 'células de posta' (del inglés, guide-post cells) para los axones de las células mitrales y empenachadas [16]. Estas células se han identificado por medio del anticuerpo monoclonal Lot1, como una subpoblación de neuronas de generación temprana que se forman, exclusivamente, en el neocórtex y que, posteriormente, migran hasta alcanzar su posición final en el telencéfalo basal, y enmarcan el área del TOL $[75,76]$. Estas células Lot1-positivas podrían producir factores que, a su vez, atrajeran o promovieran el crecimiento axonal. Se ha sugerido que, en otras células de posta, laminina y fibronectina pudieran ser sus efectores [77]. De hecho, las células Lot1-positivas parecen formar un límite en la mayoría de las estructuras del sistema olfativo más allá del cual los axones del TOL no pueden crecer [75]. Pero, la adición del Lot1 en cultivos organotípicos no modifica la formación del TOL [75]. Dado el escaso grado de heterogeneidad esperado en el TOL en comparación con el NO, parece más plausible que estos mecanismos mediados por contacto contribuyesen menos a la formación del TOL que en el caso del NO $[65,78]$.

\section{Factores secretables}

Parece muy probable que factores secretables se involucren en la formación del TOL [79]. El primero en identificarse, Slit-2, podría producirse por el tabique del cerebro anterior y actuar a través de uno de sus receptores naturales, Robo-1 [80-82]. Según ciertas evidencias, su papel en la formación del TOL no 


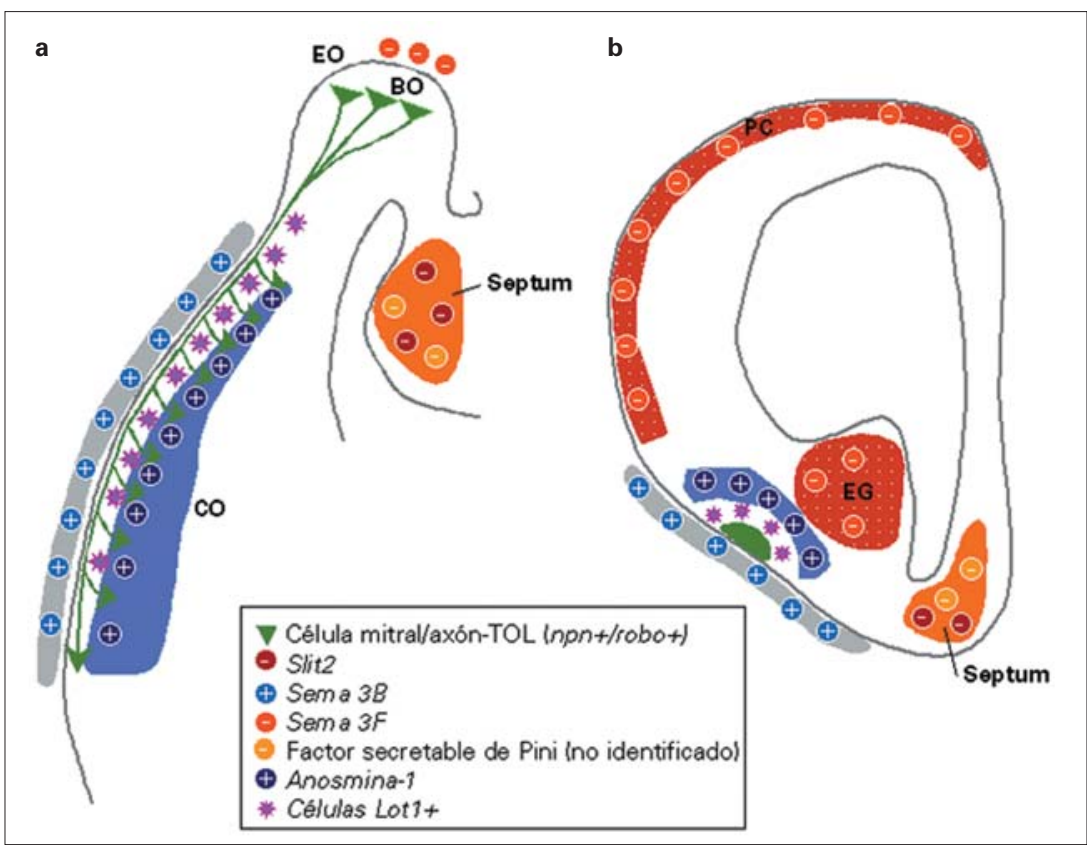

Figura 2. Factores que orientan el crecimiento de los axones de las neuronas de proyección del BO. Secciones horizontal (A) y coronal (B) de la cabeza, que muestran las estructuras olfativas y anexos. Las células mitrales y sus axones (TOL) se representan en forma de triángulos. Diferentes factores quimiorrepelentes evitan que los axones del TOL invadan distintas estructuras (septum, EG y PC) y los fuerzan a ocupar una posición en la superficie de la vesícula telencefálica. A ello coadyuvan las células lot1 positivas (estrellas) y dos factores quimioatrayentes, la Sema 3B (secretada por los progenitores de los huesos del cráneo) y anosmina1 (producida por la CO). Esta última es fundamental para la formación de colaterales a partir de los axones primarios del TOL (flechas en A), que invadirán sus dianas corticales (para detalles, ver texto). BO: bulbo olfativo; CO: corteza olfativa; EG: eminencia ganglionar; EO: epitelio olfativo; PC: placa cortical (modificado de [107]).

debería ser indispensable [40,76]. El grupo de Pini ha sugerido que debe existir una molécula concurrente y diferente de Slit-2 que fuera, de hecho, la responsable de la actividad repelente del tabique [83] (Fig. 2), aunque experimentos posteriores parecen adjudicar ese papel al efecto coordinado y conjunto de Slit-1 y 2 [82]. Resulta interesante el hecho de que la doble mutación de Slit-1 y Slit-2 produzca el desflecado de los axones del TOL, que desaparecería como tal, y proyecte los axones mitrales a regiones mucho más ventrales y menos laterales del córtex olfativo de lo que lo hacen en condiciones normales, inclusive invadiendo el tabique y llegando hasta casi la línea media, aunque sin evidencias de que consigan atravesarla y proyectar al BO contralateral [82]. Los axones del TOL responden también de forma selectiva a algunas semaforinas secretadas. Sema $3 F$ repele los axones del TOL, evita que invadan la placa cortical y la eminencia ganglionar, mientras que la Sema 3B atrae los axones del TOL, y los fuerza a permanecer en la superficie del telencéfalo [17] (Fig. 2). Por su parte, otras semaforinas secretadas, como la Sema 3A, no parecen influir en absoluto en el crecimiento de los axones del BO [17,80]. Resulta interesante que, dentro del BO, algunas células y sus axones expresan Neuropilina-1 y $2[6,31,40,84]$, los receptores funcionales de la familia de las semaforinas. En cualquier caso, los únicos mutantes nulos analizados hasta la fecha, el mutante nulo para Sema $3 \mathrm{~A}$ y el de su receptor, Neuropilina-1, no muestran alteraciones significativas en el desarrollo del TOL, aunque sí de otras estructuras olfativas [85,86]. Los receptores de los diversos miembros de la familia de las netrinas, tales como DCC o Unc5H3, se expresan fuertemente en las células mitrales del BO durante el desarrollo $[87,88]$; pero, ni Netrina-1 ni una forma secretada de Netrina-
G1 - una molécula de esta familia anclada a membrana plasmática- ejercen efecto alguno en el crecimiento de los axones del BO [17, 80,89].

Se ha sugerido que las células mitrales que proyectan al córtex olfativo deberían establecer un mapa estereotópico de proyecciones, dependiendo de los receptores odoríferos que proyectan, a su vez, a las células mitrales [21]. De hecho, las NSO que expresan el receptor odorífero I7 forman sinapsis con las células mitrales que inervan el glomérulo I7 y los axones de éstas, a su vez, deberían ordenarse formando clusters sinápticos en el córtex piriforme. Las colaterales axónicas se forman a partir de los axones primarios del TOL. Este fenómeno es una estrategia muy extendida entre los axones durante su período de navegación para invadir sus regiones diana durante el desarrollo del sistema nervioso [90], amén de ser la única utilizada por las células mitrales y las empenachadas del BO para colonizar las regiones diana olfativas en el córtex cerebral in vivo [91]. Se acepta generalmente que los mismos factores involucrados en la orientación del crecimiento axonal deberían ser los responsables de la formación de colaterales axónicas y de guiarlas hasta las neuronas adecuadas. Un ejemplo al respecto lo tenemos en Slit-2, un quimiorrepelente para axones bien conocido que parece ser un regulador positivo de la formación de colaterales a partir de axones de neuronas sensoriales [76,92], así como en las dendritas de neuronas piramidales del córtex [93]. Por el contrario, otros estudios sugieren que ambos procesos -inducción-orientación del crecimiento de colaterales axónicas y navegación de los axones- deberían gobernarse por factores diferentes [91]. Las células lot1-positivas, que no parece que participen en la formación de colaterales de las células mitrales del $\mathrm{BO}$, podrían representar un ejemplo de ello [75]. La proteína Anosmina-1 -deficiente en el síndrome de Kallmann, asociación clínica de anosmia e hipogonadismo hipogonadotrópico- parece involucrarse, directamente, en la formación de colaterales de las células mitrales durante el período del desarrollo en que el córtex olfativo se coloniza por las primeras colaterales del TOL [71]. Tras una espera de unos dos días en el ratón [91], posiblemente para facilitar la neutralización de factores inhibidores del crecimiento axonal presentes en el córtex olfativo, tal y como sugiere el grupo de Fujisawa [75], Anosmina-1 promueve el fenómeno de sprouting axonal sin afectar el crecimiento de los axones primarios (rata E17). Por el contrario, en etapas anteriores del desarrollo (rata E15), Anosmina-1 contribuye a orientar el crecimiento de los axones primarios del BO, pero no influye en el sprouting [71]. Confirmando, de alguna forma, que las moléculas responsables de la formación de colaterales axónicos no tienen forzosamente que ser las mismas que determinen el crecimiento de sus axones primarios, los patrones de expresión de las diferentes moléculas de la familia Slit no sugieren que éstas puedan desempeñar algún papel en la formación de colaterales a partir de los axones del TOL e invadir el córtex olfativo [94], y tampoco in vivo o in vitro se tiene evidencia alguna de que los 
Slit modulen realmente la formación de colaterales del TOL [82]. Tampoco las neurotrofinas se ha visto que influyan en la formación del TOL [95].

Tomado todo ello en conjunto, podría parecer que las señales que guían el crecimiento de los axones del BO actúan en cooperación: señales atrayentes y repelentes, probablemente de acuerdo con algún tipo de jerarquía, tal y como se ha sugerido [96]. Se destaca el hecho de que algunas de las moléculas que no parecen relevantes a la hora de guiar los axones de las células mitrales del BO (Sema 3A, Netrina-1), lo sean para el crecimiento de los axones de las NSO [86,97-99]. Así, parece que los papeles desempeñados por estas moléculas durante el desarrollo del circuito olfativo deberían ser diversos y, por supuesto, todavía existe la posibilidad de que factores que atraen a los axones de las NSO, pero repelan los de las células mitrales, puedan identificarse. Finalmente, debemos recordar que la interacción de moléculas secretadas con componentes no difusibles de la matriz extracelular podría tener importantes implicaciones funcionales para el establecimiento de una correcta conectividad sináptica durante el desarrollo [40,71].

Tal y como se ha descrito, hace relativamente poco, parece que los factores que guían los axones de las NSO hasta formar las sinapsis correctas dentro del BO se preservan a lo largo de toda la vida del animal $[64,65]$. Se destaca también el hecho de que los factores involucrados en la formación del TOL se presentan y expresan de forma correcta en estructuras del ratón mutante Small Eye [6,31]. Esto explicaría que se forme una estructura que recuerda al TOL en estos mutantes, a partir del ectópico y malformado OBLS [6,31]. Estos estudios enfatizan la idea de que el $\mathrm{BO}$ es independiente $\mathrm{y}$, por tanto, la existencia de un protomapa durante el desarrollo temprano del sistema olfativo. En cualquier caso, no debemos olvidar que, entre todas las señales que hemos revisado en este trabajo, tanto para la neurogénesis de las células mitrales del $\mathrm{BO}$ como para la formación inicial del TOL, el papel de Tbr-1 parece crítico [29] (Tabla).

\section{CONCLUSIONES}

Tomando en consideración todos estos datos, concluimos que la hipótesis del protomapa resulta más plausible que la teoría del protocórtex a la hora de explicar el desarrollo del sistema olfativo. Frente a un número creciente de estudios que así lo avalan, apenas unos pocos trabajos soportan el modelo del protocórtex para el desarrollo inicial. El BO se organiza de forma independiente con respecto a las proyecciones aferentes de las NSO o de otras influencias externas y, todo ello, a partir de un foco de neurogénesis en la zona más dorsorrostral del tubo neural, donde se producen las primeras neuronas de proyección del mismo, las células mitrales. En etapas posteriores, las señales derivadas de las aferencias de las NSO y, eventualmente, también otro tipo de señales contribuirían a conferir al BO su apariencia madura, tal y como lo reconocemos en el adulto, con inclusión, por supuesto, de la llegada de las interneuronas desde la zona subventricular del cerebro anterior, que derivan del telencéfalo ventral [100, 101]. En el mismo sentido, los estudios en el mutante Pax-6 su- gieren fuertemente que el establecimiento inicial de las proyecciones centrales del BO ocurre de forma independiente de los axones de las NSO que están de camino desde el EO. Estos experimentos abren la posibilidad de identificar señales moleculares intrínsecas que se requieren para la formación correcta de las conexiones del sistema olfativo. Así, y aunque es algo que todavía se ha estudiado relativamente poco, planteamos la hipótesis de que el desarrollo independiente del córtex olfativo sigue la predicción de la hipótesis del protomapa. De hecho, estudios realizados en otras regiones del cerebro apoyan, fuertemente, la idea de que un protomapa gobierna el desarrollo de la corteza piriforme. Por ejemplo, en el mutante nulo para $G b x$-2, la diferenciación talámica se interrumpe y los axones talámicos no inervan el córtex. Aún así, muchos marcadores específicos de regiones corticales se desarrollan normalmente, lo que sugiere que son factores intrínsecos del neocórtex los responsables del desarrollo de la corteza cerebral o, cuando menos, de la expresión de todos estos marcadores [102,103]. Es más, si se expresa de forma ectópica -en el telencéfalo posterior, antes de la llegada de los axones talámicos-el potente morfógeno Fgf8, provoca que el córtex somatosensorial se duplica parcialmente y una población adicional de axones talámicos se desarrolla para inervar estos barriles extra [2]. Asimismo, estudios del desarrollo de embriones mutantes con una reducción en el nivel de Fgf8, sugieren la posibilidad de que la regionalización molecular neocortical y la llegada de aferentes talámicos son procesos independientes durante el desarrollo embrionario [104].

Se ha observado también que factores intrínsecos del propio córtex durante el desarrollo, como, por ejemplo, la llegada de las fibras talamocorticales, controlan la especificación de diferentes áreas corticales [105]. Una extensión de la hipótesis del protomapa nos lleva a nosotros a considerar la expresión regional en otras estructuras telencefálicas en ausencia de sus aferencias. En este contexto, se ha aceptado de forma general que el desarrollo y diferenciación del BO depende de la llegada de los axones sensoriales olfativos. Mutantes homocigotos Small Eye no tienen estructuras nasales, con inclusión del EO, mientras que tan sólo desarrollan un BO emergente, condicionado más por un defecto en la migración de las neuronas posmitóticas que por un fallo de neurogénesis en sí mismo. Por tanto, no parece que el desarrollo inicial del BO sea dependiente de la llegada de las fibras nerviosas desde las NSO.

Estos estudios sugieren que un análisis más profundo de la formación del protomapa en las diferentes estructuras que componen el córtex olfativo podría resultar extremadamente interesante y esclarecedor. Resultaría particularmente importante para determinar las bases moleculares que gobiernan la diferenciación de la corteza olfativa LOT specific synaptic clusters que se han identificado recientemente por el grupo de Buck [21]. También podría ser interesante para llegar a ser capaces de analizar la relevancia de la llegada de las aferentes sensoriales a la hora de refinar y ultimar el desarrollo del BO. Finalmente, un modelo con un BO, pero sin TOL, podría resultar determinante a la hora de clarificar la verdadera influencia que esta proyección tiene en el desarrollo de las estructuras corticales. 
1. Rakic P. Specification of cerebral cortical areas. Science 1988; 241: 170-6.

2. Fukuchi-Shimogori T, Grove EA. Neocortex patterning by the secreted signaling molecule FGF8. Science 2001; 294: 1071-4.

3. O'Leary DD. Do cortical areas emerge from a protocortex? Trends Neurosci 1989; 12: 400-6.

4. Jefferis GS, Marin EC, Stocker RF, Luo L. Target neuron prespecification in the olfactory map of Drosophila. Nature 2001; 414: 204-8.

5. Wang X, Gao C, Norgren RB Jr. Cellular interactions in the development of the olfactory system: an ablation and homotypic transplantation analysis. J Neurobiol 2001; 49: 29-39.

6. Jiménez D, García C, de Castro F, Chédotal A, Sotelo C, De Carlos JA, et al. Evidence for intrinsic development of olfactory structures in Pax6 mutant mice. J Comp Neurol 2000; 428: 511-26.

7. Cajal SR. Origen y terminación de las fibras nerviosas olfatorias. Barcelona: Gac San; 1890.

8. Greer CA. Structural organization of the olfactory system. In Getchell TV, Doty RL, Bartoshuk LM, Snow JB Jr, eds. Smell and taste in health and disease. New York: Raven; 1991. p. 65-81.

9. Malnic B, Hirono J, Sato T, Buck LB. Combinatorial receptor codes for odors. Cell 1999; 96: 713-23.

10. Zhang X, Firestein S. The olfactory receptor gene superfamily of the mouse. Nat Neurosci 2002; 5: 124-33.

11. Mombaerts P, Wang F, Dulac C, Chao SK, Nemes A, Mendelsohn M, et al. Visualizing an olfactory sensory map. Cell 1996; 87: 675-86.

12. Valverde F. Studies on the piriform lobe. Cambridge, Mass.:Harvard University Press; 1965.

13. Price JL. A study of complementary laminar patterns of termination of afferent fibers to the olfactory cortex. J Comp Neurol 1973; 150: 87-108.

14. Devor M. Fiber trajectories of olfactory bulb efferents in the hamster. J Comp Neurol 1976; 166: 31-48.

15. Schwob JE, Price JL. The development of axonal connections in the central olfactory system of rats. J Comp Neurol 1984; 223: 177-202.

16. López-Mascaraque L, De Carlos JA, Valverde F. Early onset of the rat olfactory bulb projections. Neuroscience 1996; 70: 255-66.

17. De Castro F, Hu L, Drabkin H, Sotelo C, Chédotal A. Chemoattraction, chemorepulsion of olfactory bulb axons by different secreted semaphorins. J Neurosci 1999; 19: 4428-36.

18. Derer P, Caviness VS Jr, Sidman RL. Early cortical histogenesis in the primary olfactory cortex of the mouse. Brain Res 1977; 123: 27-40.

19. Luskin MB, Price JL. The distribution of axon collaterals from the olfactory bulb and the nucleus of the horizontal limb of the diagonal band to the olfactory cortex demonstrated by double retrograde labeling techniques. J Comp Neurol 1982; 209: 249-63.

20. Scott JW, McBride RL, Schneider SP. The organization of projections from the olfactory bulb to the piriform cortex and olfactory tubercle in the rat. J Comp Neurol 1980; 194: 519-34.

21. Zou Z, Horowitz LF, Montmayeur JP, Snapper S, Buck LB. Genetic tracing reveals a stereotyped sensory map in the olfactory cortex. Nature 2001; 414: 173-9.

22. Goldman SA, Luskin MB. Strategies utilized by migrating neurons of the postnatal vertebrate forebrain. Trends Neurosci 1998; 21: 107-14.

23. Stout RP, Graziadei PP. Influence of the olfactory placode on the development of the brain in Xenopus laevis (Daudin). I. Axonal growth and connections of the transplanted olfactory placode. Neuroscience 1980; 5: 2175-86.

24. Byrd CA, Burd GD. The quantitative relationship between olfactory axons and mitral/tufted cells in developing Xenopus with partially deafferented olfactory bulbs. J Neurobiol 1993; 24: 1229-42.

25. Graziadei PPC, Monti-Graziadei GA. The influence of the olfactory placode on the development of the telencephalon in Xenopus laevis. Neuroscience 1992; 46: 617-29.

26. Brunjes P, Frazier LL. Maturation and plasticity in the olfactory system of vertebrates. Brain Res 1986; 396: 1-45.

27. Gong Q, Shipley MT. Evidence that pioneer olfactory axons regulate telencephalon cell cycle kinetics to induce the formation of the olfactory bulb. Neuron 1995; 14: 91-101.

28. LaMantia AS, Bhasin N, Rodees K, Heemskerk J. Mesenchymal/ epithelial induction mediates olfactory pathway formation. Neuron 2000; 28: 411-25.

29. Bulfone A, Wang F, Hevner R, Anderson S, Cutforth T, Chen S, et al. An olfactory sensory map develops in the absence of normal projection neurons or GABAergic interneurons. Neuron 1998; 21: 1273-82.

30. Puelles L, Kuwana E, Puelles E, Bulfone A, Shimamura K, Keleher J, et al. Pallial, subpallial derivatives in the embryonic chick, mouse telencephalon, traced by the expression of the genes Dlx-2, Emx-1, Nkx2.1, Pax-6, and Tbr-1. J Comp Neurol 2000; 424: 409-38.
31. Nomura T, Osumi N. Misrouting of mitral cell progenitors in the Pax6/ small eye rat telencephalon. Development 2004; 131: 787-96.

32. Stoykova A, Gruss P. Roles of Pax-genes in developing and adult brain as suggested by expression patterns. J Neurosci 1994; 14: 1395-412.

33. De Carlos JA, López-Mascaraque L, Valverde F. The telencephalic vesicles are innervated by olfactory placode-derived cells: a possible mechanism to induce neocortical development. Neuroscience 1995; 68: 1167-78.

34. De Carlos JA, López-Mascaraque L, Valverde F. Early olfactory fiber projection and cell migration into the rat telencephalon. Int J Develop Neurosci 1996; 14: 853-66.

35. Bayer SA. 3H-thymidine-radiographic studies of neurogenesis in the rat olfactory bulb. Exp Brain Res 1983; 50: 329-40.

36. Roskams AJ, Cai X, Ronnett GV. Expression of neuron-specific betaIII tubulin during olfactory neurogenesis in the embryonic and adult rat. Neuroscience 1998; 83: 191-200.

37. Bailey MS, Puche AC, Shipley MT. Development of the olfactory bulb: evidence for glia-neuron interactions in glomerular formation. J Comp Neurol 1999; 415: 423-48.

38. Treloar H, Tomasiewicz H, Magnuson T, Key B. The central pathway of primary olfactory axons is abnormal in mice lacking the N-CAM180 isoform. J Neurobiol 1997; 32: 643-58.

39. Hinds JW. Autoradiographic study of histogenesis in the mouse olfactory bulb. I. Time of origin of neurons and neuroglia. J Comp Neurol 1968; 134: 287-304.

40. Sugisaki N, Hirata T, Naruse I, Kawakami A, Kitsukawa T, Fujisawa H. Positional cues that are strictly localized in the telencephalon induce preferential growth of mitral cell axons. J Neurobiol 1996; 29: 127-37.

41. Valverde F, Santacana M. Development and early postnatal maturation of the primary olfactory cortex. Brain Res Dev Brain Res 1994; 80: 96-114.

42. Ebrahimi FA, Chess A. Olfactory neurons are interdependent in maintaining axonal projections. Curr Biol 2000; 10: 219-22.

43. Humphrey T. Correlations between the development of the hippocampal formation and the differentiation of the olfactory bulbs. Ala J Med Sci 1966; 3: 235-69.

44. Yoshida M, Suda Y, Matsuo I, Miyamoto N, Takeda N, Kuatani S, et al. Emx1 and Emx2 functions in development of dorsal telencephalon. Development 1997; 124 101-11.

45. Long JE, Garel S, Depew MJ, Tobet S, Rubenstein JL. DLX5 regulates development of peripheral and central components of the olfactory system. J Neurosci 2003; 15: 568-78.

46. Guillemot F, Lo LC, Johnson JE, Auerbach A, Anderson DJ, Joyner AL. Mammalian achaete-scute homolog 1 is required for the early development of olfactory and autonomic neurons. Cell 1993; 75: 463-76.

47. Murray RC, Navi D, Fesenko J, Lander AD, Calof AL. Widespread defects in the primary olfactory pathway caused by loss of Mash1 function. J Neurosci 2003; 23: 1769-80.

48. Tisay KT, Bartlett PF, Key B. Primary olfactory axons form ectopic glomeruli in mice lacking p75NTR. J Comp Neurol 2000; 428: 656-70.

49. Sullivan SL, Bohm S, Ressler KJ, Horowitz LF, Buck LB. Target-independent pattern specification in the olfactory epithelium. Neuron 1995; 15: 779-89.

50. Konzelmann S, Saucier D, Strotmann J, Breer H, Astic L. Decline and recovery of olfactory receptor expression following unilateral bulbectomy. Cell Tissue Res 1998; 294: 421-30.

51. Fan J, Ngai J. Onset of odorant receptor gene expression during olfactory sensory neuron regeneration. Dev Biol 2001; 229: 119-27.

52. Benson TE, Ryugo DK, Hinds JW. Effects of sensory deprivation on the developing mouse olfactory system: a light and electron microscopic morphometric analysis. J Neurosci 1984; 4: 638-53.

53. Meisami E. Effects of olfactory deprivation on postnatal growth of the rat olfactory bulb utilizing a new method for production of neonatal unilateral anosmia. Brain Res 1976; 107: 437-44.

54. Brunjes PC. Unilateral naris closure and olfactory system development. Brain Res Rev 1994; 19: 146-60.

55. Couper-Leo JM, Devine AH, Brunjes PC. Focal denervation alters cellular phenotypes and survival in the developing rat olfactory bulb. $\mathrm{J}$ Comp Neurol 2000; 417: 325-36.

56. Frazier LL, Brunjes PC. Unilateral odor deprivation: early postnatal changes in olfactory bulb cell density and number. J Comp Neurol 1988; 269: 355-70.

57. Matsutani S, Yamamoto N. Differentiation of mitral cell dendrites in the developing main olfactory bulbs of normal and naris-occluded rats. J Comp Neurol 2000; 418: 402-10.

58. Meisami E, Safari L. A quantitative study of the effects of early unilateral olfactory deprivation on the number and distribution of mitral and 
tufted cells and of glomeruli in the rat olfactory bulb. Brain Res 1981; 221: 81-107.

59. Cummings DM, Emge DK, Small SL, Margolis FL. Pattern of olfactory bulb innervation returns after recovery from reversible peripheral deafferentation. J Comp Neurol 2000; 421: 362-73.

60. Lin DM, Wang F, Lowe G, Gold GH, Axel R, Ngai J, et al. Formation of precise connections in the olfactory bulb occurs in the absence of odorant-evoked neuronal activity. Neuron 2000; 26: 69-80.

61. Belluscio L, Gold GH, Nemes A, Axel R. Mice deficient in G (olf) are anosmic. Neuron 1998; 20: 69-81.

62. Buiakova OI, Baker H, Scott JW, Farbman A, Kream R, Grillo M, et al. Olfactory Marker Protein (OMP) gene deletion causes altered physiological activity of olfactory sensory neurons. Proc Natl Acad Sci USA 1996; 93: 9858-63.

63. Zheng C, Feinstein P, Bozza T, Rodríguez I, Mombaerts P. Peripheral olfactory projections are differentially affected in mice deficient in a cyclic nucleotide-gated channel subunit. Neuron 2000; 26: 81-91.

64. Gogos JA, Osborne J, Nemes A, Mendelsohn M, Axel R. Genetic ablation and restoration of the olfactory topographic map. Cell 2000; 103: 609-20.

65. Potter SM, Zheng C, Koos DS, Feinstein P, Fraser SE, Mombaerts P. Structure and emergence of specific olfactory glomeruli in the mouse. J Neurosci 2001; 21: 9713-23.

66. Hogan BL, Horsburgh G, Cohen J, Hetherington CM, Fisher G, Lyon MF. Small eyes (Sey) a homozygous lethal mutation on chromosome 2 which affects the differentiation of both lens and nasal placodes in the mouse. J Embryol Exp Morphol 1986; 97: 95-110.

67. Stoykova A, Fritsch R, Walther C, Gruss P. Forebrain patterning defects in Small eye mutant mice. Development 1996; 122: 3453-65.

68. Bartolomei JC, Greer CA. The organization of piriform cortex and the lateral olfactory tract following the loss of mitral cells in PCD mice. Exp Neurol 1998; 154: 537-50.

69. Seki T, Arai Y. Expression of highly polysialylated NCAM in the neocortex, piriform cortex of the developing and the adult rat. Anat Embryol (Berl) 1991; 184: 395-401.

70. Stoeckli ET, Landmesser LT. Axonin-1 Nr-CAM and Ng-CAM play different roles in the in vivo guidance of chick commissural neurons. Neuron 1995; 14: 1165-79.

71. Soussi-Yanicostas N, de Castro F, Julliard K, Perfettini I, Chédotal A, Petit C. Anosmin-1 defective in the X-linked form of Kallmann syndrome promotes axonal branch formation from olfactory bulb output neurons. Cell 2002; 109: 217-28.

72. Caviness VS Jr, Sidman RL. Olfactory structures of the forebrain in the reeler mutant mouse. J Comp Neurol 1972; 145: 85-104.

73. St John JA, Key B. Chemically and morphologically identifiable glomeruli in the rat olfactory bulb. J Comp Neurol 2001; 436: 497-507.

74. Pasterkamp RJ, Peschon JJ, Spriggs MK, Kolodkin AL. Semaphorin 7A promotes axon outgrowth through integrins and MAPKs. Nature 2003; 424: 398-405.

75. Sato Y, Hirata T, Ogawa M, Fujisawa H. Requirement for early-generated neurons recognized by monoclonal antibody lot 1 in the formation of lateral olfactory tract. J Neurosci 1998; 18: 7800-10.

76. Hirata T, Fujisawa H, Wu JY, Rao Y. Short-Range Guidance of Olfactory Bulb Axons Is Independent of Repulsive Factor Slit. J Neurosci 2001; 21: 2373-9.

77. Kuhn TB, Schmidt MF, Kater SB. Laminin and fibronectin guideposts signal sustained but opposite effects to passing growth cones. Neuron 1995; 14: 275-85.

78. Treloar HB, Feinstein P, Mombaerts P, Greer CA. Specificity of glomerular targeting by olfactory sensory axons. J Neurosci 2002; 22: 2469-77.

79. Pini A. Chemorepulsion of axons in the developing mammalian central nervous system. Science 1993; 261: 95-8.

80. Li HS, Chen JH, Wu W, Fagaly T, Zhou L, Yuan W, et al. Vertebrate slit a secreted ligand for the transmembrane protein roundabout is a repellent for olfactory bulb axons. Cell 1999; 96: 807-18.

81. Nguyen Ba-Charvet KT, Brose K, Marillat V, Kidd T, Goodman CS, Tessier-Lavigne M, et al. Slit2-mediated chemorepulsion and collapse of developing forebrain axons. Neuron 1999; 22: 1-20.

82. Nguyen-Ba-Charvet KT, Plump AS, Tessier-Lavigne M, Chedotal A. Slit1 and slit2 proteins control the development of the lateral olfactory tract. J Neurosci 2002; 22: 5473-80.

83. Patel K, Nash JA, Itoh A, Liu Z, Sundaresan V, Pini A. Slit proteins are not dominant chemorepellents for olfactory tract and spinal motor axons. Development 2001; 128: 5031-7.
84. Kawakami A, Kitsukawa T, Takagi S, Fujisawa H. Developmentally regulated expression of a cell surface protein neuropilin in the mouse nervous system. J Neurobiol 1996; 29: 1-17.

85. Kitsukawa T, Shimizu M, Sanbo M, Hirata T, Taniguchi M, Bekku Y, et al. Neuropilin-semaphorin III/D-mediated chemorepulsive signals play a crucial role in peripheral nerve projection in mice. Neuron 1997; 19: 995-1005.

86. Taniguchi M, Nagao H, Takahashi YK, Yamaguchi M, Mitsui S, Yagi T, et al. Distorted odor maps in the olfactory bulb of Semaphorin 3A-deficient mice. J Neurosci 2003; 23: 1390-97.

87. Przyborski SA, Knowles BA, Ackerman SL. Embryonic phenotype of Unc5h3 mutant mice suggests chemorepulsion during the formation of the rostral cerebellar boundary. Development 1998; 125: 41-50.

88. Shu T, Valentino KM, Seaman C, Cooper HM, Richards LJ. Expression of the netrin-1 receptor deleted in colorectal cancer (DCC) is largely confined to projecting neurons in the developing forebrain. J Comp Neurol 2000; 416: 201-12.

89. Nakashiba T, Ikeda T, Nishimura S, Tashiro K, Honjo T, Culotti JG, et al. Netrin-G1: a novel glycosyl phosphatidylinositol-linked mammalian netrin that is functionally divergent from classical netrins. J Neurosci 2000; 20: 6540-50.

90. Kalil K, Szebenyi G, Dent EW. Common mechanisms underlying growth cone guidance and axon branching. J Neurobiol 2000; 44: 145-58.

91. Hirata T, Fujisawa H. Environmental control of collateral branching and target invasion of mitral cell axons during development. J Neurobiol 1999; 38: 93-104.

92. Wang KH, Brose K, Arnott D, Kidd T, Goodman CS, Henzel W, et al. Biochemical purification of a mammalian slit protein as a positive regulator of sensory axon elongation and branching. Cell 1999; 96: 771-84

93. Whitford KL, Marillat V, Stein E, Goodman CS, Tessier-Lavigne M, Chedotal A, et al. Regulation of cortical dendrite development by SlitRobo. Neuron 2002; 33: 47-61.

94. Marillat V, Cases O, Nguyen Ba-Charvet KT, Tessier-Lavigne M, Sotelo C, Chedotal A. Spatiotemporal expression patterns of slit and robo genes in the rat brain. J Comp Neurol 2002; 442: 130-55.

95. Hirata T, Fujisawa H. Cortex-specific distribution of membrane-bound factors that promote neurite outgrowth of mitral cells in culture. J Neurobiol 1997; 32: 415-25.

96. Stein E, Tessier-Lavigne M. Hierarchical organization of guidance receptors: silencing of netrin attraction by slit though Robo/DCC receptor complex. Science 2001; 291: 1928-38

97. Renzi MJ, Wexler TL, Raper JA. Olfactory sensory axons expressing a dominant-negative semaphorin receptor enter the CNS early and overshoot their target. Neuron 2000; 28: 437-47.

98. Williams-Hogarth LC, Puche AC, Torrey C, Cai X, Song I, Kolodkin $\mathrm{AL}$, et al. Expression of semaphorins in developing and regenerating olfactory epithelium. J Comp Neurol 2000; 423: 565-78.

99. Schwarting GA, Raitcheva D, Crandall JE, Burkhardt C, Puschel AW. Semaphorin 3A-mediated axon guidance regulates convergence, targeting of P2 odorant receptor axons. Eur J Neurosci 2004; 19: 1800-10.

100. Luskin MB. Restricted proliferation and migration of postnatally generated neurons derived from the forebrain subventricular zone. Neuron 1993; 11: 173-89.

101. Lois C, Álvarez-Buylla A. Long-distance neuronal migration in the adult mammalian brain. Science 1994; 264: 1145-8.

102. Donoghue MJ, Rakic P. Molecular evidence for the early specification of presumptive functional domains in the embryonic primate cerebral cortex. J Neurosci 1999; 19: 5967-79.

103. Miyashita-Lin EM, Hevner R, Wassarman KM, Martínez S, Rubenstein JL. Early neocortical regionalization in the absence of thalamic innervation. Science 1999; 285: 906-9.

104. Garel S, Huffman KJ, Rubenstein JL. Molecular regionalization of the neocortex is disrupted in Fgf8 hypomorphic mutants. Development 2003; 130: 1903-14.

105. Schlaggar BL, O'Leary DD. Potential of visual cortex to develop an array of functional units unique to somatosensory cortex. Science 1991; 252: $1556-60$

106. Hardelin JP, Julliard AK, Moniot B, Soussi-Yanicostas N, Verney C, Schwanzel-Fukuda M, et al. Anosmin-1 is a regionally restricted component of basement membranes and interstitial matrices during organogenesis: implications for the developmental anomalies of X chromosome-linked Kallmann syndrome. Dev Dyn 1999; 215: 26-44.

107. López-Mascaraque L, de Castro F. The olfactory bulb as an independent developmental domain. Cell Death Differ 2002; 9: 1279-86. 
PROTOCÓRTEX FRENTE A PROTOMAPA: UNA PERSPECTIVA DESDE EL BULBO OLFATIVO

Resumen. Introducción y objetivo. El sistema olfativo es un modelo extraordinario para la investigación de la guía y la conectividad del crecimiento axonal. Durante el desarrollo, el epitelio olfativo, el bulbo olfativo y la corteza olfativa diferencian varios tipos celulares, y extienden sus proyecciones axonales. Dado que hay una relación estrecha entre estas tres estructuras, nos hacemos la siguiente pregunta: ¿es necesaria la llegada de las aferencias sensoriales procedentes del epitelio olfativo para iniciarse la formación de las proyecciones centrales del bulbo olfativo? Esto nos lleva a otra pregunta más general: ¿es necesario el establecimiento de conexiones aferentes para inducirse un programa de desarrollo en las células diana? Desarrollo. El establecimiento inicial de las proyecciones centrales del bulbo olfativo ocurre independientemente de la llegada de los axones del epitelio olfativo, lo que refuerza la idea de que las diferentes regiones corticales se predeterminan ya antes de la migración de neuronas posmitóticas, por lo menos en el caso del bulbo olfativo. Esto implica un control molecular, intrínseco y estricto de las distintas estructuras del sistema olfativo. Conclusiones. Entonces, ¿cómo encuentran las proyecciones axonales su correcta localización dentro del cerebro? Mecanismos por contacto y moléculas quimiotrópicas cooperan para fijar su posición en el telencéfalo, y evitan que los axones del bulbo invadan otras estructuras diferentes a la corteza olfativa. Al mismo tiempo, estimulan la formación de colaterales axónicas, en una orquesta de señales atrayentes/permisivas y repulsivas/inhibidoras. En etapas posteriores del desarrollo, se completará la apariencia madura del bulbo olfativo. [REV NEUROL 2004; 39: 146-55]

Palabras clave. Desarrollo. Guía axonal. Mutantes. Neurogénesis. Olfacción. Telencéfalo.

\section{PROTOCÓRTEX VERSUS PROTOMAPA: UMA PERSPECTIVA SOB O BOLBO OLFACTIVO}

Resumo. Introdução e objectivo. O sistema olfactivo é um modelo extraordinário para a investigação da guia e da conectividade do crescimento axonal. Durante o desenvolvimento, o epitélio olfactivo, o bolbo olfactivo e o córtex olfactivo diferenciam diversos tipos celulares, estendendo as suas projecções axonais. Dado que existe uma relação estreita entre estas três estruturas, colocámo-nos a seguinte pergunta: é necessária a chegada das aferências sensoriais procedentes do epitélio olfactivo para ter início a formação das projecções centrais do bolbo olfactivo? Isto conduz-nos a outra pergunta ainda mais geral: é necessário o estabelecimento de conexões aferentes para induzir-se um programa de desenvolvimento nas células alvo? Desenvolvimento. O estabelecimento inicial das projecções centrais do bolbo olfactivo ocorre independentemente da chegada do axónios do epitélio olfactivo, reforçando a ideia de que as diferentes regiões corticais se predeterminam já antes da migração de neurónios pós-mióticos, pelo menos no caso do bolbo olfactivo. Isto envolve um controlo molecular, intrínseco e restrito das distintas estruturas do sistema olfactivo. Conclusões. Então, como encontram as projecções axoniais a sua localização correcta dentro do cérebro? Mecanismos por contacto e moléculas quimiotrópicas cooperam para fixar a sua posição no telencéfalo, e evitam que os axónios do bolbo invadam outras estruturas diferentes do córtex olfactivo. Ao mesmo tempo, estimulam a formação de colaterais axónicas, e uma orquestra de sinais atraentel permissivas e repulsivas/inibidoras. Em etapas posteriores do desenvolvimento, completar-se-á a aparência madura do bolbo olfactivo. [REV NEUROL 2004; 39: 146-55]

Palavras chave. Desenvolvimento. Guia axonal. Mutantes. Neurogénese. Olfacto. Telencéfalo. 